\title{
Denoising Approaches Using Fuzzy Logic and Convolutional Autoencoders for Human Brain MRI Image
}

Nishant Chauhan and Byung-Jae Choi

Department of Electrical Engineering, Daegu University, Gyeongsan, Korea

\section{]jfis}

\begin{abstract}
In biomedical images, one of the serious issues is noise which affects their coherent nature. To analyze the results for the detection and treatment of disease, it is essential to remove the noise. The advancement in brain imaging technologies requires reasonable techniques for pre-processing steps like denoising, deblurring, contrast enhancement, etc. Magnetic resonance imaging (MRI) images of the human brain are often corrupted with noises due to the application of various image acquisition techniques, operator performance, and types of equipment. In this paper, we evaluate several fuzzy logic based denoising filters. A combined approach of fuzzy logic and a convolutional autoencoder has been also used on a brain image dataset for the performance evaluation. The experimental results show that the combined approach performs better than other methods.
\end{abstract}

Keywords: MRI images, Fuzzy logic, Autoencoders, Denoising, PSNR

Received: Jun. 7, 2019

Revised : Jun. 21, 2019

Accepted: Sep. 20, 2019

This research was supported(in part) by the Daegu University Research Grant.

Correspondence to: Byung-Jae Choi (bjchoi@daegu.ac.kr)

(CThe Korean Institute of Intelligent Systems

cCThis is an Open Access article distributed under the terms of the Creative Commons Attribution Non-Commercial License (http://creativecommons.org/licenses/ by-nc/3.0// which permits unrestricted noncommercial use, distribution, and reproduction in any medium, provided the original work is properly cited.

\section{Introduction}

Medical imaging such as X-rays, computed tomography (CT), and magnetic resonance imaging (MRI) are susceptible to noise. The reason behind is physical source which causes the thermal fluctuations in the receiver or sometimes the limitations of equipment. MRI images usually suffers from Gaussian noise, salt $\&$ pepper noise, Poisson noise and speckle noise. This increases the requirement for an appropriate denoising technique for good image analysis.

Based on different characteristics, various denoising methods have been proposed. An image registration method called PORTR (pre-operative and post-recurrence brain tumor registration) was introduced which removes the pathological regions from the image-based processing term after completion of the mapping of the images [1]. It also estimates the overlap between aligned and resection. Some techniques based on non-local means filter use averaging the similar image pixels according to their intensity distance.

Block-matching and 3D filtering (BM3D) denoising method collects the group patches from similar reference patches to construct a 3D array [2]. Similar denoising performance can be achieved using multi-layer perception (MLP) model. Wavelet transformation method denoises the images by holding all the signal characteristics irrespective of its frequency [3, 4]. The wavelet filter removes the noisy artifacts from the signal. But it also filtered those pixels/structures, whose size are similar to noisy pixels which is a major drawback.

Fuzzy techniques have been already applied in many domain of image processing such as 
filtering, image enhancement, etc. The fuzzy based filtering is basically considered as nonlinear filtering method [5]. The advantage is to detect the uncertainty of noise and removing that noise by forming fuzzy rules and membership function generated by understanding the image properties [6].

The recent research on image denoising with the development of deep convolutional neural network has shown a progressive approach with highly improved results [7]. The results based on deep neural architectures are encouraging with the recent evolution in deep learning. Autoencoders for image denoising showed better performance than conventional denoising methods [8]. An autoencoder is a type of artificial neural network that tries to learn an approximation of the input using identity function with the help of back propagation algorithms. An autoencoder model aims to learn a representation of set of data (encoding), commonly for dimensionality, by training the model to ignore signal noise. Using convolutional layer in autoencoders enhances the performance for their ability to exploit strong spatial correlations.

In this paper we evaluate the performance of several denoising approaches of fuzzy filtering and convolutional denoising autoencoding (CDAE) and their combination on MRI images of the human brain [9, 10]. The performance comparison has been done using PSNR (Peak Signal to Noise Ratio) [11].

\section{Denoising Approaches}

\subsection{Fuzzy Filtering}

Linear filtering methods are insufficient in noise reduction if the noise has abnormal distribution in the image. Linear filtering methods have limited impact in noise reduction. Therefore, a need of non-linear noise filters are highly desirable. The main idea behind the fuzzy filter is to average a pixel value using other neighborhood pixel values [6]. If the input image is $x(i, j)$, the output of the fuzzy filter is can be calculated by Eq. (1):

$$
y(i, j)=\frac{\sum_{(r, s) \in A} F[x(i+r, j+s)] \cdot x(i+r, j+s)}{\sum_{(r, s) \in A} F[x(i+r, j+s)]},
$$

where, $F[x(i, j)]$ and $A$ are a window function and the area of the window, respectively. For $N \times N$ window, the range of $r$ and $s$ are $-R \leq r \leq R$ and $-S \leq s \leq S$, respectively, where $N=2 R+1=2 S+1$. We use the gaussian fuzzy filter, in which the center value of the window is a median. In this case

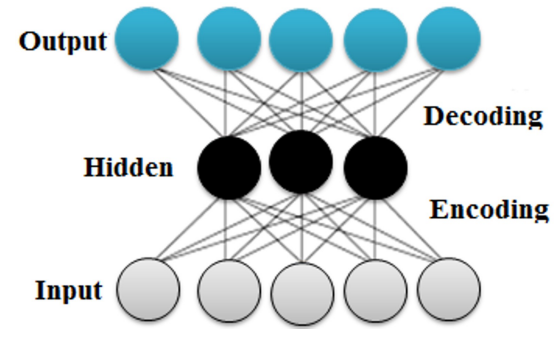

Figure 1. Basic structure of autoencoder.

the window function can be defined by Eq. (2):

$$
F[x(i+r, j+s)]=e^{\frac{1}{2}\left[\frac{x(i+r, j+s)-x_{\operatorname{med}}(i, j)}{\sigma(i, j)}\right]^{2}},
$$

where $x_{\text {med }}(i, j)$ and $\sigma(i, j)$ are a median value and a variance value for all the input values $x(i+r, j+s)$ for $r, s \in A$, respectively.

Practically, if the width of a window is small $(N=3)$, the preservation of edges is maximum. Generally, for low level of noise the low window width is suitable however in case of high level noise larger window width should be considered.

\subsection{Convolutional Denoising Autoencoder}

An autoencoder is a self-supervised neural network in which the input and output are supposed to be same. An autoencoder takes input and encode it to hidden representation. These hidden representation decoded back to generate an output. The output should have the same shape as input. A basic structure of an autoencoder has been shown in Figure 1.

In Figure 1, the first layer is input layer, second layer called hidden layer which stores the hidden representation of input data (encoding) and final layer is output layer (decoding). One of the main characteristics of autoencoder is that if the number of hidden layers is less than the input layers, then the model maps the compressed approximation. This process is similar to Principal Component Analysis (PCA) which is restricted to linear map. However, an autoencoder uses non linear activation function and multiple layers which enable it to learn non-linear transformation.

A convolutional autoencoder is based on a basic autoencoder architecture having convolutional encoding and decoding layers. In the architecture the weights are shared among all input location. The feature map for a mono channel input can be defined by Eq. (3):

$$
h^{i}=s\left(x * W^{i}+b^{i}\right),
$$


where $*$ and $s$ denote the convolution and an activation function, respectively. And the reconstruction of encoded images is obtained by Eq. (4):

$$
y=s\left(\sum_{i \in H} h^{i} * W^{i}+c\right),
$$

where $c$ and $H$ are a bias per input and a feature map group, respectively. A back-propagation algorithm is used for calculation of gradient of the error function.

\subsection{Combined Approach}

A combination approach of fuzzy filtering and convolutional autoencoder method has been used for the denoising of human brain MRI images. Firstly, the noisy brain MRI images have been denoised by fuzzy filtering and then the resulting images are used as test images for pretrained convolutional denoising autoencoder (CDAE). The final image has a superior quality as compared to separate algorithm results.

\section{Experimental Results}

In this paper, a dataset of human brain MRI Lesion Segmentation in Multiple Sclerosis has been used which is freely available on e-health laboratory webpage, Department of Computer Science, University of Cyprus [12]. It has 1,600 human brain MRI images. The sample test images have been shown in Figure 2.

For the experiment, Gaussian noise and Poisson noise have been used to corrupt the images. The noise parameters have been given in Table 1. Here, $p$ is a proportion of noise introduced, $\sigma$ and $\mu$ are standard deviation and mean of normal distribution, respectively. $\lambda$ is a mean of Poisson distribution sample of noisy images. It has been shown in Figure 3.
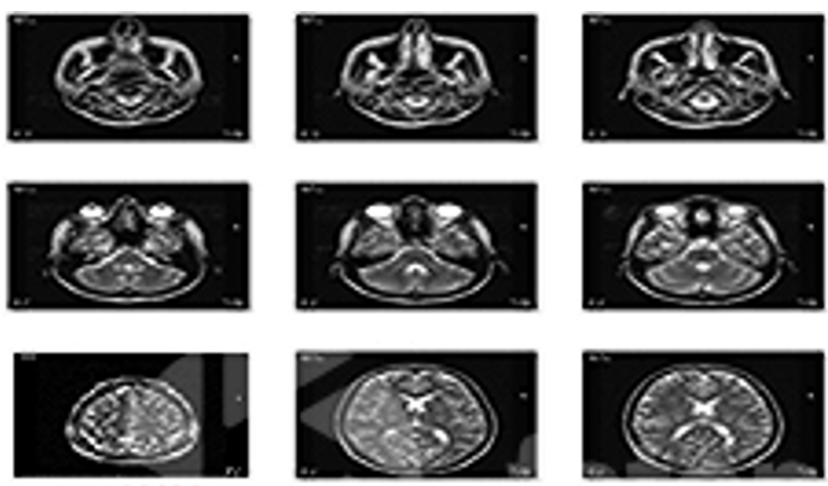

Figure 2. Randomly chosen sample images from dataset.
Table 1. Induced noise parameters

\begin{tabular}{cc}
\hline Noise type & Corrumption values \\
\hline Gaussian & $p=0.1, \sigma=1, \mu=0$ \\
Possion & $p=0.1, \lambda=1$ \\
\hline
\end{tabular}
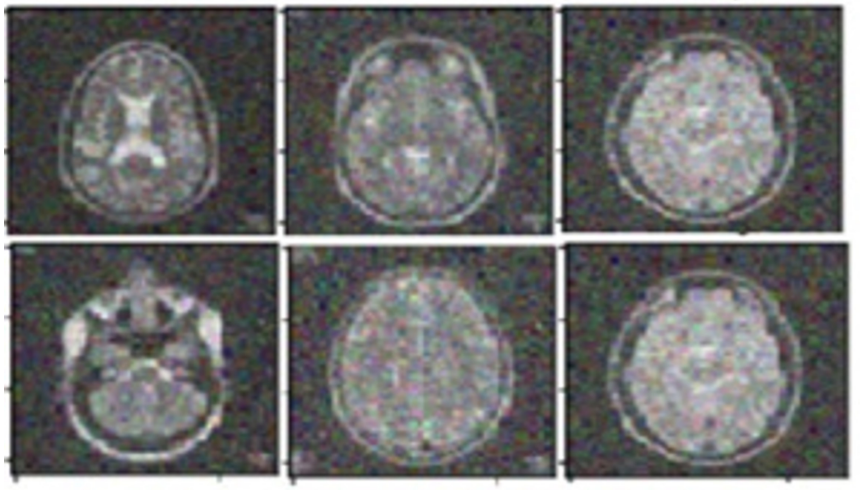

Figure 3. Randomly chosen sample noisy images.

In Figure 3, images contain high Gaussian and Poission noises. A fuzzy filtering has been applied on 50 randomly selected images and these 50 images are used as a test images.

An architecture for the CDAE model has been shown in Figure 4.

All images are pre-processed for training and two different criteria of training has been used. In first case, all images have been resized to $64 \times 64$, with batch size $=5$ and the model has been trained with training rate $=0.00$ for 100 epochs.

In second case, all images have been resized to $128 \times 128$, with batch size $=8$ and with same training rate for 100 epochs. The model has been implemented using Keras and OpenCV libraries on Samsung PC (Intel Core i5-4590, 16 GB RAM, Nvidia GEFORCE GTX 1070 Ti GPU). The denoising performance has been compared by using PSNR calculation.

The denoising results using CDAE and combination of fuzzy filtering and CDAE are presented in Figures 5 and 6, respectively.

As shown in Figure 5, the performance of CDAE in high and complex noise is impressive but it has some blurred effect on high contrast regions. However, the combined approach reflects good visual results because the convolution mapped the important features of training images in encoding process and it considers the very important details of the image features while generating denoised image. In Figure 6, test image resolution is $(128 \times 128)$ however if it still has the blurry effect in important regions of MRI image. 


\begin{tabular}{|c|c|c|}
\hline \multirow{2}{*}{ Input_1(InputLayer). } & Input.1 & (None, $1,64,64)$ \\
\hline & Output. & (None, $1,64,64)$ \\
\hline \multicolumn{3}{|l|}{$\downarrow$} \\
\hline \multirow{2}{*}{ Convolution2d_1(Convolution2D). } & Input.? & (None, $1,64,64)$ \\
\hline & Output. & (None, $64,64,64)$. \\
\hline \multicolumn{3}{|l|}{$\downarrow$} \\
\hline \multirow{2}{*}{ Maxpooling2d_1(MaxPooling2D). } & Input.? & (None, $64,64,64$ ). \\
\hline & Output. & (None, $64,32,32)$. \\
\hline \multicolumn{3}{|l|}{$\downarrow$} \\
\hline \multirow{2}{*}{ Convolution2d_2(Convolution2D). } & Input.? & (None, $64,32,32)$. \\
\hline & Output. & (None, $64,32,32)$. \\
\hline$\downarrow$ & & \\
\hline \multirow{2}{*}{ Maxpooling2d_2(MaxPooling2D). } & Input.? & (None, $64,32,32$ ). \\
\hline & Output. & (None,64,16,16). \\
\hline$\downarrow$ & & \\
\hline \multirow{2}{*}{ Convolution2d_3(Convolution2D). } & Input.. & (None,64,16,16). \\
\hline & Output. & (None,64,16,16). \\
\hline$\downarrow$ & & \\
\hline \multirow{2}{*}{ Upsampeling2d_1(UpSampeling2D) } & Input.: & (None, $64,16,16$ ). \\
\hline & Output. & (None, $64,32,32)$ \\
\hline \multicolumn{3}{|l|}{$\downarrow$} \\
\hline \multirow{2}{*}{ Convolution2d_4(Convolution2D). } & Input.? & (None, 64,32,32). \\
\hline & Output. & (None,64,32,32). \\
\hline$\downarrow$ & & \\
\hline \multirow{2}{*}{ Upsampeling2d_2(UpSampeling2D). } & Input. & (None, 64,32,32). \\
\hline & Output. & (None,64,64,64). \\
\hline \multicolumn{3}{|l|}{$\downarrow$} \\
\hline \multirow{2}{*}{ Convolution2d_5(Convolution2D). } & Input.1 & (None, $64,64,64)$. \\
\hline & Output. & (None, $1,64,64)$ \\
\hline
\end{tabular}

Figure 4. Model architecture of CDAE.
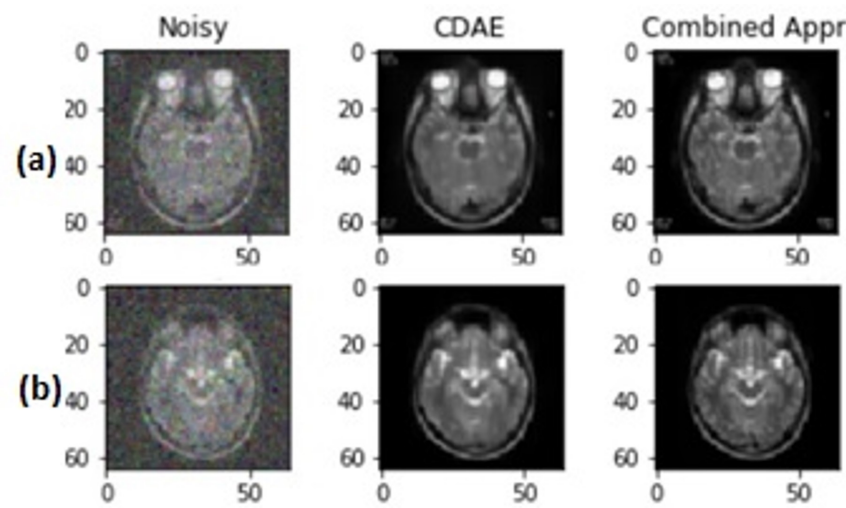

Figure 5. Denoising results using fuzzy filtering and combined approach $((64 \times 64), 100$ epochs $)$.

Training and validation loss curves for both cases have been presented in Figure 7.
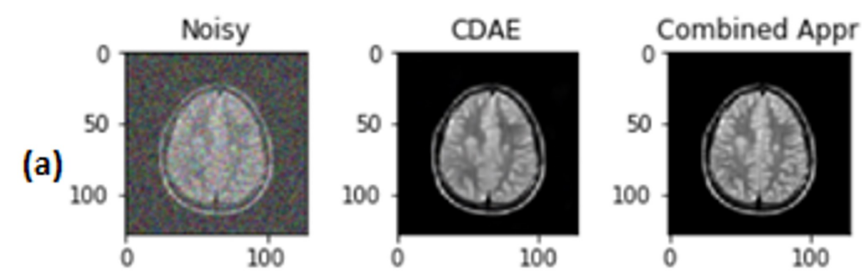

(b)

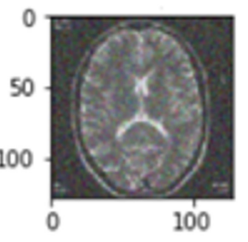

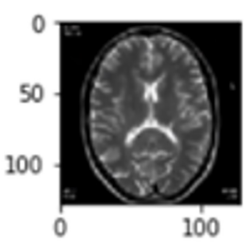

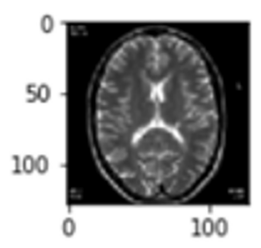

Figure 6. Denoising results using fuzzy filtering and combined approach $((128 \times 128), 100$ epochs $)$.

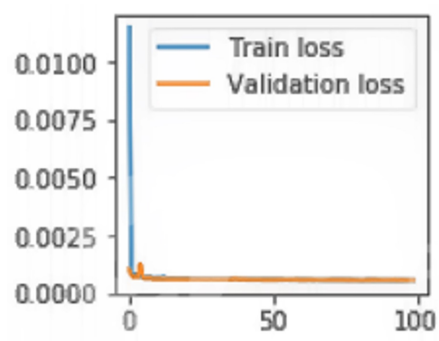

(a)

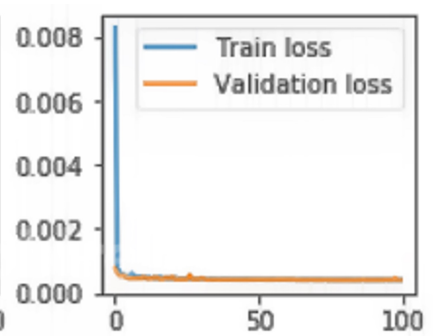

(b)
Figure 7. Validation land training loss for $($ a) $(64 \times 64), 100$ epochs, (b) $(128 \times 128), 100$ epochs.

Table 2. Comparison of PSNR for fuzzy filtering and combined approach denoising methods

\begin{tabular}{ccc}
\hline Images & CDAE & Combined approach \\
\hline Figure 5(a) & 17.16 & 19.20 \\
\hline Figure 5(b) & 16.41 & 17.23 \\
\hline Figure 6(a) & 21.30 & 23.17 \\
Figure 6(b) & 20.81 & 23.87 \\
\hline
\end{tabular}

Training loss is an error of the training set of data and validation loss is an error of running the validity data on the network after training. If the resolution increase, CDAE can map more valuable features and give better results even if the number of epochs are less (Figure 7(b)).

As demonstrated in Table 2, the combined approach of fuzzy logic and CDAE can be useful for brain MRI image denoising.

\section{Conclusion}

The combined performance of these two techniques also very powerful and can be effective in diagnosing the abnormilities in MRI images. Low resolution images can be denoised remark- 
ably by training CDAE model with high epochs and suitable learning rate.. Therefore we can conclude that the combination approach has better performance than individual algorithms.

\section{References}

[1] D. Kwon, M. Niethammer, H. Akbari, M. Bilello, C. Davatzikos, and K. M. Pohl, "PORTR: pre-operative and post-recurrence brain tumor registration," IEEE Transactions on Medical Imaging, vol. 33, no. 3, pp. 651-667, 2013. http://doi.org/10.1109/TMI.2013.2293478

[2] K. Dabov, A. Foi, V. Katkovnik, and K. Egiazarian, "Image denoising by sparse 3-D transform-domain collaborative filtering," IEEE Transactions on Image Processing, vol. 16, no. 8, pp. 2080-2095, 2007. http://doi.org/10.1109/ TIP.2007.901238

[3] J. Portilla, V. Strela, M. J. Wainwright, and E. P. Simoncelli, "Image denoising using scale mixtures of Gaussians in the wavelet domain," IEEE Transactions on Image Processing, vol. 12, no. 11, pp. 1338-1351, 2003. http://doi.org/10.1109/TIP.2003.818640

[4] R. R. Coifman and D. L. Donoho, Translation-Invariant Denoising. New York, NY: Springer, 1995.

[5] H. Q. Zhang, X. J. Ma, and N. Wu, "A new filter algorithm of image based on fuzzy logical," in Proceedings of 2011 International Symposium on Computer Science and Society, Kota Kinabalu, Malaysia, 2011, pp. 315-318. http://doi.org/10.1109/ISCCS.2011.91

[6] H. K. Kwan, "Fuzzy filters for noisy image filtering," in Proceedings of the 2003 International Symposium on Circuits and Systems, Bangkok, Thailand, 2003. http: //doi.org/10.1109/ISCAS.2003.1205798

[7] Y. H. Cho, "A study on diagnosis of brain tumor based on neural network by extracting valid area and texture features by GLCM of MRI image," Journal of the Korean Institute of Intelligent Systems, vol. 27, no. 3, pp. 261-267, 2017. http://doi.org/10.5391/JKIIS.2017.27.3.261

[8] L. Gondara, "Medical image denoising sing convolutional denoising autoencoders," in Proceedings of IEEE 16th International Conference on Data Mining Workshops, Barcelona, Spain, 2016, pp. 241-246. http://doi.org/10. 1109/ICDMW.2016.0041
[9] S. B. Lee and Y. H. Cho, "A diagnostic assistant system development of brain subarachnoid hemorrhage by deep learning of CT images," Journal of the Korean Institute of Intelligent Systems, vol. 28, no. 1, pp. 71-76, 2018. http://doi.org/10.5391/JKIIS.2018.28.1.71

[10] H. S. Hwang and J. S. Oh, "Evolutionary design of morphology-based homomorphic filter for feature enhancement of medical images," International Journal of Fuzzy Logic and Intelligent Systems, vol. 9, no. 3, pp. 172177, 2009. https://doi.org/10.5391/IJFIS.2009.9.3.172

[11] A. Hore and D. Ziou, "Image quality metrics: PSNR vs. SSIM," in Proceedings of 2010 20th International Conference on Pattern Recognition, Istanbul, Turkey, 2010, pp. 2366-2369. http://doi.org/10.1109/ICPR.2010.579

[12] MRI Lesion Segmentation in Multiple Sclerosis Database, Available http://www.ehealthlab.cs.ucy.ac.cy/idex.php/ facilities/32-software/218-datasets

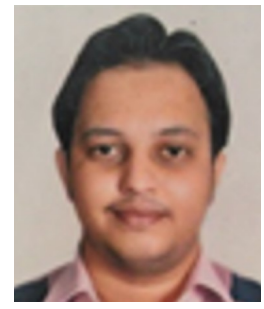

Nishant Chauhan received his B.Tech. degree degree in computer science and engineering at Noida Institute of Engineering and Technology from Dr. A.P.J. Abdul Kalam Technical University, India in 2012. He is a graduate student (master course) at Daegu University majoring Control Measurement. His research interests include intelligent control, fuzzy logic, and image processing.

E-mail: nishantsep1090@daegu.ac.kr.

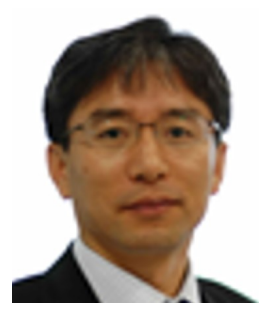

Byung-Jae Choi received his B.S. in Electronic Engineering from Kyungpook National University, Daegu, Korea, in 1987. And he received his M.S. and Ph.D. degrees in Electrical and Electronic Engineering, KAIST, Daejeon, Korea, in 1989 and 1998, respectively. Since 1999, he is a Professor of School of Electronic and Electrical Engineering, Daegu University, Daegu, Korea. His current research interests include intelligent control and its applications.

E-mail: bjchoi@daegu.ac.kr. 